\title{
Stent-Assisted Balloon-Induced Intimal Disruption and Relamination in Aortic Dissection Repair: The STABILISE concept
}

\author{
Sophie C. Hofferberth, MBBS, BSc, ${ }^{a}$ Ian K. Nixon, MBBS, FRACS, ${ }^{a}$ Raymond C. Boston, PhD, ${ }^{b}$ \\ Craig S. McLachlan, PhD, MPH, ${ }^{\mathrm{c}}$ and Peter J. Mossop, MBBS, FRACR ${ }^{\mathrm{d}, \mathrm{e}}$
}

\begin{abstract}
Objectives: The study objective was to describe the Stent-Assisted Balloon-Induced Intimal Disruption and Relamination in Aortic Dissection Repair technique for aortic dissection repair using proximal descending aortic endografting with distal aortic relamination through bare-metal stent and balloon-induced intimal disruption with immediate intimal reapposition.
\end{abstract}

\begin{abstract}
Methods: Between April 2007 and September 2011, 11 selected patients (10 male; median age, 50 years) underwent proximal descending aortic endografting plus stent-assisted balloon-induced intimal disruption of the thoracoabdominal aorta to treat complicated aortic dissection (7 type A, 4 acute type B). Patients with type A dissection underwent open surgical intervention plus adjunctive retrograde endovascular repair. Serial computed tomography angiography was used to assess aortic remodeling.
\end{abstract}

Results: There were no intraprocedural complications. Thirty-day incidence of death, stroke, and paralysis/visceral ischemia was $9 \%(\mathrm{n}=1), 0 \%$, and $0 \%$, respectively. Median follow-up was 18 months (range, 4-54 months). Two patients $(18 \%)$ required secondary endovascular reintervention. No late adverse events or aortic-related deaths occurred. Complete false lumen obliteration occurred in $90 \%(\mathrm{n}=10)$ of patients, with stable maximal diameters in the thoracic $(P=.6)$ and abdominal aortas (celiac trunk: $P=.34$; renal; $P=.6$; infrarenal: $P=.7$ ) at latest follow-up.

Conclusions: The Stent-Assisted Balloon-Induced Intimal Disruption and Relamination in Aortic Dissection Repair approach is a feasible endovascular technique that shows promise to achieve complete repair of the dissected aorta by inducing complete false lumen obliteration. The restoration of uniluminal flow in the thoracoabdominal aorta has the potential to improve long-term outcomes. Prospective, multicenter investigations are required to implement this strategy more broadly. (J Thorac Cardiovasc Surg 2014;147:1240-5)

The last decade has seen the emergence of endovascular therapies to treat descending aortic dissection. ${ }^{1,2}$ Standard endovascular repair currently consists of stent-graft coverage of the proximal entry tear in type $\mathrm{B}$ dissection. ${ }^{1-6}$ Recent studies report the use of hybrid open surgery with descending aortic stent grafting in type A dissection. ${ }^{7-11}$ The aim of proximal stent-graft coverage of the primary entry tear is to induce thoracic false lumen thrombosis and promote aortic remodeling. However, deficiencies

From the Department of Cardiac Surgery, ${ }^{a}$ St Vincent's Hospital, Melbourne,

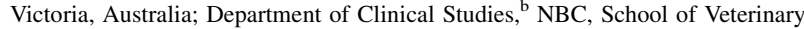
Medicine, University of Pennsylvania, Philadelphia, Pa; Rural Clinical School, ${ }^{\mathrm{c}}$ Faculty of Medicine, University of New South Wales, Kensington, Australia; Department of Medical Imaging, ${ }^{\mathrm{d}}$ St Vincent's Hospital, Melbourne, Victoria, Australia; and Cardiovascular Research Centre, ${ }^{\mathrm{e}}$ Australian Catholic University, Melbourne, Victoria, Australia

Disclosures: I.K.N. and P.J.M. were involved in patenting the Zenith Dissection stent. Both receive a share of patent royalties from Cook, Inc. All other authors have nothing to disclose with regard to commercial support.

Received for publication Jan 7, 2013; revisions received March 8, 2013; accepted for publication March 19, 2013; available ahead of print April 22, 2013.

Address for reprints: Sophie C. Hofferberth, MBBS, BSc, St Vincent's Hospital Melbourne, Department of Cardiac Surgery, Level 4, Clinical Sciences Building, Fitzroy, Victoria 3065, Australia (E-mail: s.hofferberth@ugrad.unimelb.edu.au). $0022-5223 / \$ 36.00$

Copyright (c) 2014 by The American Association for Thoracic Surgery

http://dx.doi.org/10.1016/j.jtcvs.2013.03.036 persist with regard to the distal dissected aorta. An experimental model of aortic stenting performed in canines first described this phenomenon 20 years ago. ${ }^{12}$ Proximal endograft closure may result in incomplete aortic reconstruction, with a failure to remodel the abdominal aorta in up to $80 \%$ of cases. ${ }^{5}$ This risks late complications, such as aneurysmal degeneration, rupture, and distal reoperation.

Our group has pioneered the Staged Total Aortic and Branch Vessel Endovascular (STABLE) reconstruction technique, an endovascular strategy that combines proximal descending aortic endografting with distal bare-metal stenting. ${ }^{13,14}$ Midterm results suggest this approach prevents aortic growth and improves aortic remodeling, with low periprocedural morbidity. ${ }^{15,16}$ However, despite extensive distal aortic repair, $75 \%$ of patients receiving STABLE repair have some degree of false lumen perfusion at midterm follow-up. ${ }^{15,16}$ This observation prompted us to evolve the STABLE approach to a technique that would achieve false lumen elimination and immediately restore uniluminal thoracoabdominal aortic flow. We termed this the "Stent-Assisted Balloon-Induced Intimal Disruption and Relamination in Aortic Dissection Repair" (STABILISE) technique. The STABILISE approach combines proximal 


\section{Abbreviations and Acronyms \\ CTA = computed tomography angiography \\ STABILISE $=$ Stent-Assisted Balloon-Induced \\ Intimal Disruption and Relamination \\ in Aortic Dissection Repair \\ STABLE $=$ Staged Total Aortic and Branch \\ Vessel Endovascular \\ TEE $=$ transesophageal echocardiography}

descending aortic endografting with balloon-induced disruption of the distal delaminated intimal flap and simultaneous reapproximation of the intimal layer to the outer thoracoabdominal aortic wall with a dissection stent. We report the intermediate results of this novel endovascular strategy in type A and B dissection.

\section{MATERIALS AND METHODS}

A retrospective review of the cardiovascular surgical database at St Vincent's Hospital, Melbourne, was performed from 2007 to 2011. Patient medical records were reviewed for preoperative patient characteristics, dissection morphology, details of operative strategy, intraoperative events, and postoperative course.

\section{Patients}

From April 2007 to September 2011, 67 patients presented to our tertiary referral center with type A or B aortic dissection. Twenty-nine of these patients underwent endovascular treatment for repair of acute (type $\mathrm{A}=10$, type $\mathrm{B}=11$ ) and chronic (type $\mathrm{A}=4$, type $\mathrm{B}=4$ ) aortic dissection. Of these, 11 selected patients underwent the STABILISE procedure for management of acute (type $\mathrm{A}=3$, type $\mathrm{B}=4$ ) and chronic (post-type $\mathrm{A}=4$ ) aortic dissection at St Vincent's Hospital. Patient selection included malperfusion or branch vessel involvement, proximal false lumen growth ( $>5 \mathrm{~mm}$ in 12 months), or true lumen collapse (Table 1).

Additional anatomic inclusion criteria included descending thoracoabdominal aortic diameter (distal endograft landing zone) $36 \mathrm{~mm}$ or less, nonaneurysmal abdominal aorta with true lumen collapse, and no evidence of periaortic hematoma/rupture in the zone to be stented. No patients with known connective tissue disease were included in the study.

Ten patients $(91 \%)$ were male, with a median age of 50 years (range, 35-67 years) (Table 1). Preoperative patient demographics and risk factors are detailed in Table 2. The median interval from dissection diagnosis to STABILISE procedure was 2 days (range, 1-9 days) for acute disease and 308 days (range, 175-1733 days) for chronic disease.

The St Vincent's Hospital Ethics Committee approved this retrospective study and waived the need for individual patient consent. All patients gave informed consent to each procedure.

\section{Endovascular Prosthesis}

The Zenith Dissection Endovascular System (Cook Medical Inc, Bloomington, Ind) is a modular system specifically designed to treat aortic dissection, consisting of a proximal component, the Zenith TX2 TAA Endovascular Graft, and a distal component, the Zenith Dissection Endovascular Stent. A detailed description of the Zenith Dissection Endovascular System has been reported. ${ }^{14,15,17}$

\section{Procedure}

Baseline computed tomography angiography (CTA) with multiplanar reconstruction was performed to assess the feasibility of the endovascular procedure, patency, and origin (true or false lumen) of visceral vessels and cerebral circulation, choice of peripheral access site, and sizing of stent grafts.

All procedures were performed in a hybrid operating suite under general anesthesia. Patients with type A dissection underwent open proximal surgical intervention as indicated, plus adjunctive retrograde descending aortic endograft deployment with bare-metal stenting in cases in which dissection extended beyond the resected aorta. The extent of bare-metal stenting was determined by the distal extension of the dissection.

The common femoral artery was used as the peripheral access vessel in all patients. Fluoroscopy and transesophageal echocardiography (TEE) were used to guide the placement of a Lunderquist Extra Stiff Double Curved Exchange Wire Guide (Cook Medical Inc) over a pigtail catheter maneuvered in the true lumen. The proximal Zenith TX2 endograft (length range, $120-150 \mathrm{~mm}$ ) was then advanced and deployed. Endograft sizing was performed to enable an adequate seal within both the proximal neck and the distal dissected thoracic aorta and was based on the outer aortic diameter of the proximal landing zone. Coverage of the left subclavian artery was undertaken in 3 patients $(27 \%)$ to enable proximal entry tear closure after adequate collateral cerebral circulation had been confirmed. Proximal endograft coverage was extended to the thoracoabdominal aortic junction or to a distal thoracic landing site at which the descending thoracic aorta was $36 \mathrm{~mm}$ or less.

Delivery systems were then exchanged, and the Zenith Dissection Endovascular Stent (diameter, $46 \mathrm{~mm}$ ) was advanced and deployed, overlapping at least 1 stent body with the proximal endograft. The extent of aortic delamination determined the choice of Zenith Dissection Stent. Combinations of $4(82 \mathrm{~mm}), 6(126 \mathrm{~mm})$, and $8(164 \mathrm{~mm})$ body length stents were used to approximate the extent of dissection, and each overlapped by 1 stent body. Angiography confirmed there was incomplete expansion of the intimal flap with residual patency of the false lumen.

Subsequently, a Coda balloon catheter (Cook Medical Inc) was deployed via the Dissection Stent introducer sheath. After accurate positioning in the lower third of the covered stent graft, the balloon was inflated under fluoroscopic and TEE guidance within the true lumen of the distal endograft. Balloon expansion within the distal third of the stent graft was undertaken to the point of intimal flap disruption, leading to reapposition of the intima to the aortic wall. Serial balloon dilatation of the stented true lumen was undertaken in a caudal direction to propagate the intimal tear and simultaneously reoppose the intima to the aortic wall throughout the thoracoabdominal aorta (Figure 1). On completion of balloon angioplasty, angiography was performed to ensure adequate seal of the thoracic false lumen and to assess false lumen obliteration and branch vessel patency in the thoracoabdominal aorta.

Balloon sizing was based on the maximal aortic diameter at the distal landing zone. Balloon pressures ranged from 2 to 4 atmospheres with inflation to approximately $80 \%$ to $90 \%$ of total aortic size to enable intimal disruption without applying pressure to the outer aortic wall.

The STABILISE technique was modified in cases involving dissected visceral or renal branches that were considered at risk of obstruction at the time of intimal disruption. In these cases, a safety guidewire or diagnostic catheter was placed into the branch vessel once initial stenting had been completed to allow for potential access after Dissection Stent dilatation.

\section{Aortic Remodeling}

Patients were evaluated with post-procedure CTA, and serial follow-up CTA imaging was performed at 3,6 , and 12 months and annually thereafter to assess false lumen status and aortic cross-sectional diameter.

\section{Data Analysis}

Results were analyzed with SPSS version 20.0 (SPSS Inc, Chicago, Ill). Data were assessed for normality and expressed as number (\%) for categoric and median (range) for continuous variables. Two-tailed Student $t$ test was used to analyze continuous variables. 
TABLE 1. Clinical characteristics and indications for STABILISE procedure

\begin{tabular}{|c|c|c|c|c|}
\hline $\mathbf{N}$ & $\begin{array}{c}\text { Sex/age } \\
(y)\end{array}$ & Dissection type & $\begin{array}{c}\text { Time since } \\
\text { diagnosis }(d)\end{array}$ & $\begin{array}{c}\text { Indication for } \\
\text { STABILISE } \\
\text { procedure }\end{array}$ \\
\hline 1 & $\mathrm{~F} / 47$ & Acute type B & 5 & TLC, renal malperfusion \\
\hline 2 & $\mathrm{M} / 51$ & $\begin{array}{l}\text { Post type A } \\
\text { repair: acute }\end{array}$ & 1 & $\begin{array}{l}\text { Aorta-iliofemoral } \\
\text { malperfusion }\end{array}$ \\
\hline 3 & $\mathrm{M} / 67$ & Acute type B & 1 & TLC \\
\hline 4 & $\mathrm{M} / 62$ & Acute type B & 9 & $\begin{array}{l}\text { Mesenteric, renal } \\
\text { malperfusion }\end{array}$ \\
\hline 5 & $\mathrm{M} / 41$ & $\begin{array}{l}\text { Post type A } \\
\text { repair: chronic }\end{array}$ & 361 & TLC, high-flow FL \\
\hline 6 & $\mathrm{M} / 42$ & $\begin{array}{l}\text { Post type A } \\
\text { repair: chronic }\end{array}$ & 84 & Renal malperfusion \\
\hline 7 & $\mathrm{M} / 47$ & $\begin{array}{l}\text { Post type A } \\
\text { repair: acute }\end{array}$ & 1 & TLC \\
\hline 8 & $\mathrm{M} / 52$ & $\begin{array}{l}\text { Post type A } \\
\text { repair: chronic }\end{array}$ & 255 & Left common iliac TLC \\
\hline 9 & $\mathrm{M} / 52$ & $\begin{array}{l}\text { Post type A } \\
\text { repair: chronic }\end{array}$ & 1733 & $\begin{array}{l}\text { Expanding FL aneurysm, } \\
\text { high-flow FL }\end{array}$ \\
\hline 10 & $\mathrm{M} / 49$ & $\begin{array}{l}\text { Post type A } \\
\text { repair: acute }\end{array}$ & 6 & $\begin{array}{l}\text { Visceral, renal } \\
\text { malperfusion }\end{array}$ \\
\hline 11 & $\mathrm{M} / 35$ & Acute type B & 2 & $\begin{array}{l}\text { Aorta-infrarenal, limb } \\
\text { malperfusion }\end{array}$ \\
\hline
\end{tabular}

STABILISE, Stent-Assisted Balloon-Induced Intimal Disruption and Relamination in Aortic Dissection Repair; $T L C$, true lumen collapse; $F L$, false lumen.

\section{RESULTS}

\section{Procedural/Thirty-Day Outcomes}

The median number of devices (endograft plus stents) deployed per patient was 3 (range, 2-5). In 3 patients, the proximal endograft completely covered the left subclavian artery, 2 had partial coverage, and 6 had no coverage. Among the 7 patients with type A dissection, proximal open surgical repair consisted of total arch, hemiarch, or isolated aortic replacement in 1,4 , and 2 patients,

TABLE 2. Patient demographics and risk factors of 11 study patients

\begin{tabular}{ll}
\hline \multicolumn{1}{c}{ Risk factors } & No. $(\%)$ \\
\hline Age, median \pm SD & $50 \pm 9$ \\
Male & $10(91)$ \\
Dissection morphology & \\
$\quad$ Acute type A dissection & $3(28)$ \\
Chronic type A dissection & $4(36)$ \\
Acute type B dissection & $4(36)$ \\
Marfan syndrome & $1(9)$ \\
Hypertension & $9(82)$ \\
Bicuspid aortic valve & $2(22)$ \\
Diabetes & $1(9)$ \\
Active smoking & $4(44)$ \\
Peripheral vascular disease & $1(9)$ \\
Previous cardiovascular operation & $2(22)$ \\
AVR & $1(9)$ \\
Type A aortic dissection repair & $1(9)$ \\
\hline
\end{tabular}

$S D$, Standard deviation; $A V R$, aortic valve replacement.
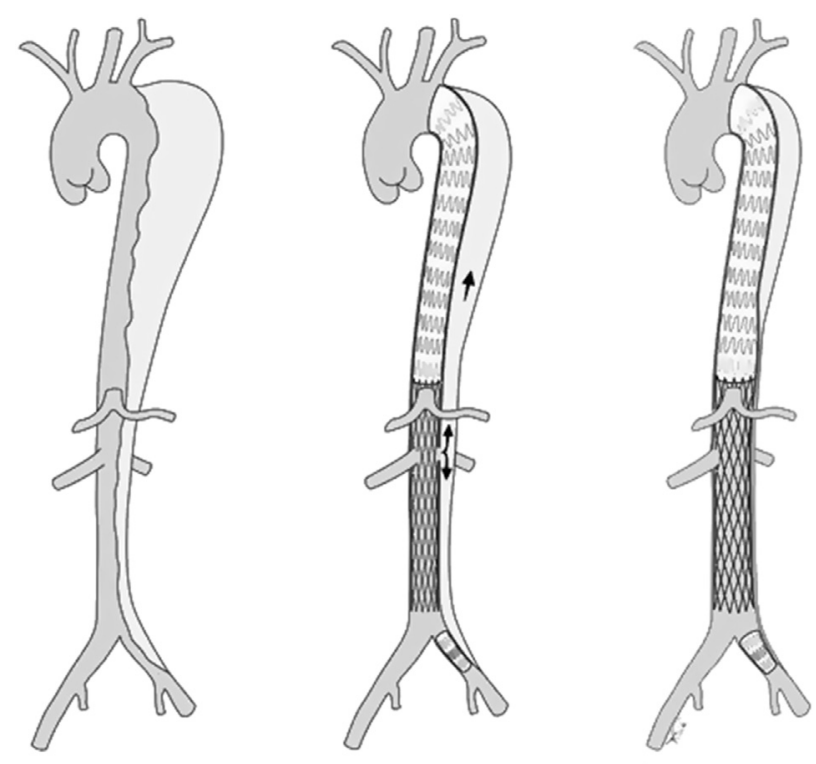

A

B

C

FIGURE 1. Schematic representation of the STABILISE repair. A, Type B aortic dissection. B, After proximal endografting with bare-metal stent implantation over the mesenteric segment with residual false lumen inflow. $\mathrm{C}$, After balloon expansion of the distal endograft and bare dissection stent to obliterate the distal dissection plane and false lumen.

respectively. Six of the 7 patients underwent concomitant aortic valve resuspension, and 1 patient required an aortic valve replacement. Three patients were noted to have supra-aortic branch vessel dissection. Median cardiopulmonary bypass and deep hypothermic circulatory arrest times were $221 \pm 28$ minutes and $30 \pm 15$ minutes, respectively.

No intraprocedural complications occurred in relation to endovascular device deployment or intimal flap disruption. There was no evidence of aortic or branch vessel injury.

There was zero incidence of postoperative stroke, spinal cord, limb, or visceral ischemia. One patient $(9 \%)$ who underwent intervention to treat renal malperfusion required transient hemofiltration after undergoing STABILISE procedure.

Two patients (18\%) required adjunctive endovascular procedures during their primary hospital stay. Both required additional stent-graft deployment to treat type 1 endoleak. A 52-year-old man with chronic type A dissection underwent further balloon dilation within the secondarily deployed stent-graft to enable adequate closure of the proximal leak. The other patient received concomitant coil embolization. Both recovered uneventfully, and follow-up CTA at 3 months demonstrated resolution of type I reentry flow.

One death $(9 \%)$ occurred within 30 days of procedure. This was a 58-year-old man who was transferred to St Vincent's Hospital 6 days after proximal surgical repair for acute type A dissection. The patient had undergone ascending aortic replacement with aortic valve resuspension. 
This patient had ongoing visceral and renal malperfusion postoperatively and was subsequently transferred emergently. He underwent a successful STABILISE procedure, with complete thoracoabdominal false lumen obliteration and restoration of visceral and renal blood flow. After an uneventful postoperative course, the patient was discharged on day 19 postprocedure. Unfortunately, the patient died unexpectedly of aortic rupture 2 weeks later. Autopsy reported localized dehiscence at the distal anastomosis site of the ascending aortic graft. The stented descending thoracic and abdominal aortas were noted to be clearly intact.

\section{Follow-up Outcomes}

Clinical follow-up was completed in all patients. The median length of follow-up was 19 months (range, 7-54 months). Overall actuarial survival was $73 \%$ at 54 months, with an aortic-specific survival of $91 \%$. There was 1 late non-aortic-related death; a 69-year-old man died 2 years after STABILISE repair secondary to bladder carcinoma. There were no late adverse events in relation to the STABILISE procedure, including zero incidence of aortic rupture, stent migration, intimal flap erosion, redissection, and branch vessel occlusion. No secondary reinterventions were required at intermediate follow-up, with no late cardiovascular events.

\section{Aortic Remodeling}

Follow-up CTA beyond 6 months was available in 9 of 10 survivors. Maximal diameters were stable in the thoracic (level of carina, $40.8 \pm 13 \mathrm{~mm}$ to $41.1 \pm 5 \mathrm{~mm}, P=.6$ ) and abdominal (level of celiac trunk, $26.6 \pm 3.8 \mathrm{~mm}$ to $28.5 \pm 5.7 \mathrm{~mm}, P=.34$; level of renal arteries: $30.3 \pm 3 \mathrm{~mm}$ to $31.3 \pm 4 \mathrm{~mm}, P=.6$; infrarenal aorta: $30.1 \pm 3 \mathrm{~mm}$ to $29.3 \pm 5.8 \mathrm{~mm}, P=.7$ ) aortas (Figure 2). In all 10 survivors $(100 \%)$, complete false lumen obliteration was achieved in the thoracic aorta, with 9 of $10(90 \%)$ achieving obliteration of the false lumen throughout the entire abdominal aorta (Figure 3).

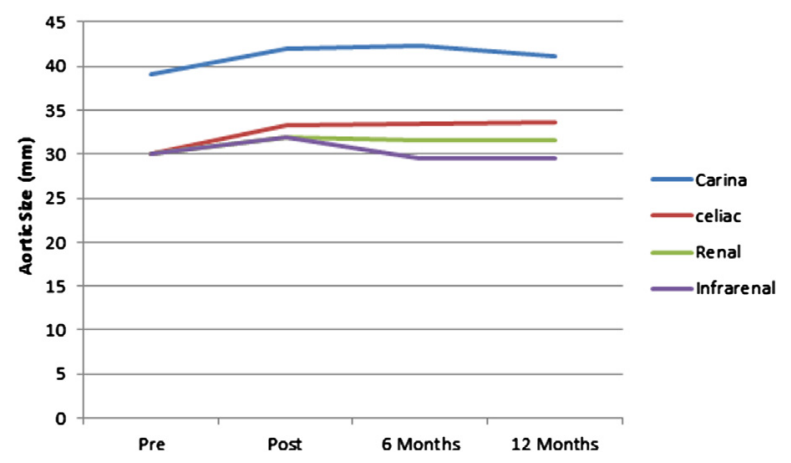

FIGURE 2. Median thoracoabdominal aortic diameters after STABILISE repair.
In 1 patient (52-year-old man treated 1733 days after initial proximal surgical repair for type A dissection), intimal disruption was only partially achieved in the abdominal aorta, with subsequent failure of complete reapposition to the aortic wall and ongoing false lumen patency. It was decided to conservatively manage this patient, while assessing for signs of aortic growth or false lumen expansion. At latest radiologic follow-up, there was no evidence of aneurysmal dilatation within the thoracic or abdominal aorta (Figure 1).

\section{DISCUSSION}

To prevent the late complications associated with incomplete distal aortic remodeling, our group has pioneered the STABLE technique, an endovascular approach combining the use of proximal descending aortic endografts with distal bare-metal stents. ${ }^{13,14}$ The rationale for bare-metal stent scaffolding in the distal dissected segment is to provide immediate support and expansion of the aortic true lumen, thereby reversing distal true lumen collapse and acting to prevent branch vessel malperfusion by reperfusing the true lumen. ${ }^{13,18}$ A number of case series have since reported the use of distal bare-metal stents for management of selected patients with type B dissection, with encouraging short-term outcomes. ${ }^{17-19}$ One-year outcomes from the multicenter STABLE trial (investigating the use of proximal TX2 thoracic stent grafts and distal bare metal dissection stents) for patients with complicated type B dissection also have demonstrated favorable clinical and anatomic outcomes. ${ }^{20}$ Our group recently published the results of our initial case series using the STABLE technique in type $\mathrm{A}$ and $\mathrm{B}$ aortic dissection. ${ }^{15}$ Midterm outcomes demonstrated low periprocedural morbidity and mortality rates, with favorable distal aortic remodeling through stent support of the distal true lumen. ${ }^{15,16}$

The STABLE treatment paradigm was based on the hypothesis that anatomic correction of the dissection would prevent the long-term sequelae associated with a residually dissected distal aorta. The STABLE approach appears to prevent distal aortic growth and enable true lumen reconstitution through endograft and stent reconstruction of the dissected aorta and branch vessels. A component of this technique is the necessity, in some cases, for staged endovascular interventions to eliminate residual reentries or enhance false lumen thrombosis. ${ }^{15,16,21}$ Although this strategy has resulted in excellent midterm outcomes ${ }^{15,16}$ additional endovascular interventions may add to procedural cost and technical complexity. To address this problem, the technique was modified in selected cases to a more simplified methodology in which the intimal flap is completely realigned to the aortic wall through balloon-induced rupture and full reexpansion by the stent scaffold.

Although this is a small study involving a selected group of inhomogeneous patients, it provides insight into the 


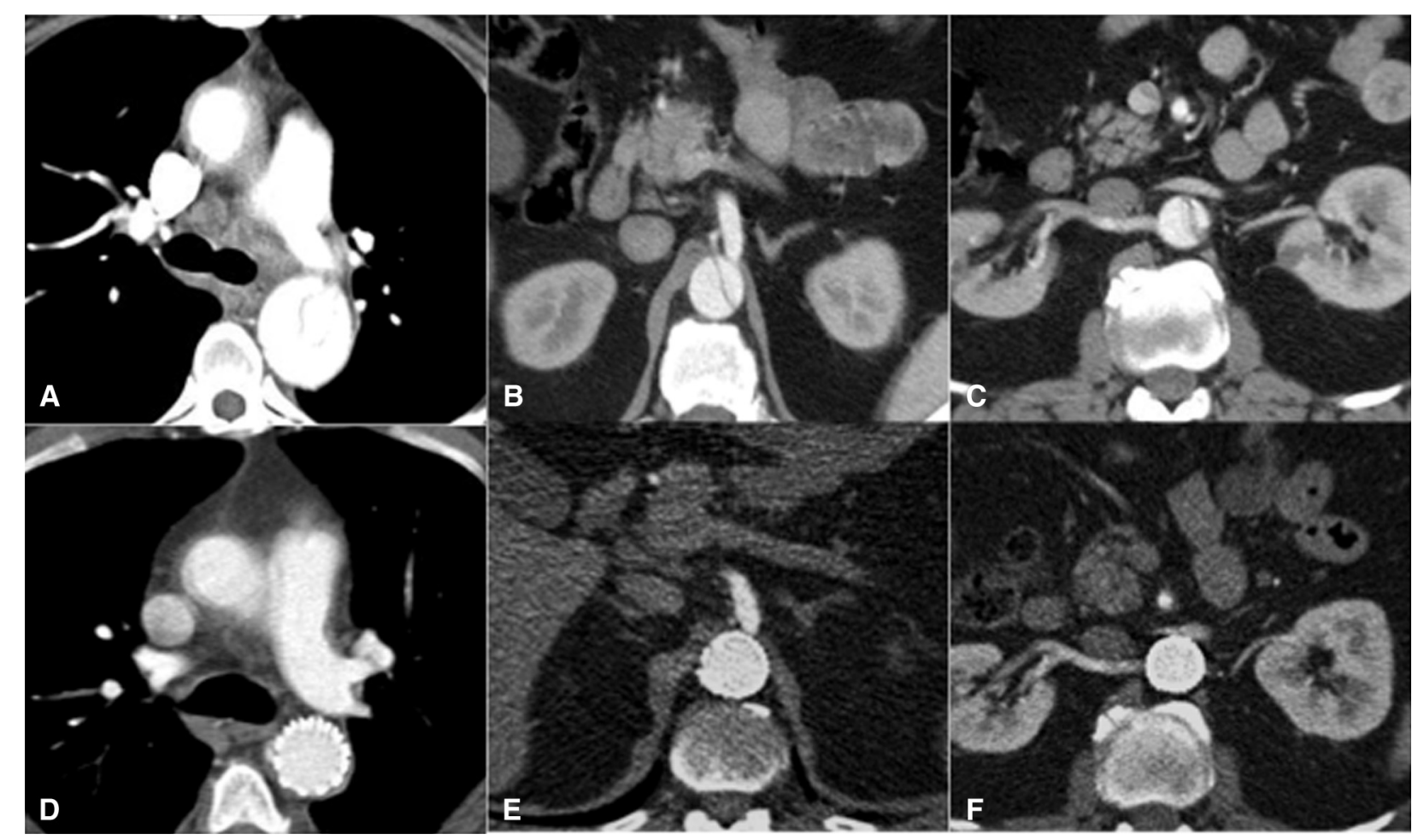

FIGURE 3. Preoperative coronal CTA scan of acute type B aortic dissection extending in a semicoronal plane at the level of the carina (A), celiac axis (B), and renal arteries $(\mathrm{C})$, with the false lumen to the right. After STABILISE remodeling of the descending thoracic aorta with restoration of normal aortic dimension (D, carina) and complete false lumen obliteration with reapposition of the intimal flap throughout the stented abdominal aorta, at the level of the celiac axis (E) and renal arteries (F), respectively.

feasibility and likely longer-term outcomes that may be achieved when the STABILISE strategy is appropriately implemented. Our early experience using this technique has demonstrated that balloon-induced intimal disruption coupled with bare-metal stent scaffolding of the distal aorta restores uniluminal flow dynamics in the thoracoabdominal aorta. In our experience, the avoidance of any potential serious complications is dependent on performing the following steps during the STABILISE procedure: (1) ensure accurate deployment of the proximal stent-graft and dissection stent in the true lumen. We depend on TEE for accurate wire placement and standard angiography to confirm true lumen placement. (2) The balloon disruption must be performed carefully using a balloon that is sized less than the outer aortic diameter. We appreciate that intuitively this seems to be an inherently risky approach; however, in our experience it has proven to be remarkably straightforward and simple to achieve intimal disruption and reapproximation throughout the distal dissected aorta. This reconstructive approach appears to prevent visceral/spinal cord malperfusion in the acute setting without increasing periprocedural morbidity. Further, at 1-year follow-up, this technique appears to stabilize thoracoabdominal aortic remodeling.

In regard to patient selection and procedural timing, STABILISE is applicable in cases in which the stented component of the aorta is nonaneurysmal. Therefore, this approach is more likely to be effective in the setting of acute dissection before distal aortic dilatation has occurred, and intimal flap to aortic wall relamination is possible. The combined use of self-expanding stents and balloon disruption of the intimal flap seems to confer safety to the procedure because intimal disruption is achieved within the aortic lumen and the outer aortic wall is not expanded by the balloon. The dissection stent predominantly functions to then reoppose the intima to the aortic wall with low radial force. Although it may be feasible to create a uniluminal aorta simply by balloon rupture of the intimal membrane, to do this without stent support would result in an unpredictable mobile intimal flap. This would also eliminate the potential to reintegrate the intima with the aortic wall achieved through stentbased reexpansion.

In cases of chronic aortic dissection, in which the intimal flap is organized or distal aneurysmal degeneration has already occurred, we believe the STABLE procedure is a more appropriate technique, with the aim of endovascular intervention being intimal flap preservation and induction of false lumen thrombosis. In our experience, intimal disruption in chronic dissection appears more difficult, which is consistent with the one case of failed intimal disruption seen in this study in a patient with chronic dissection posttype A repair. 


\section{CONCLUSIONS}

Early results demonstrate that the novel STABILISE technique is a technically feasible endovascular therapy. It achieves high rates of false lumen obliteration in aortic dissection and prevents thoracoabdominal aortic growth at intermediate follow-up. This strategy has the potential to achieve complete repair of the dissected aorta through thoracoabdominal false lumen obliteration. This may improve long-term outcomes and eliminate the need for future reintervention because more complete aortic remodeling is achieved in the acute setting. Prospective, multicenter trials are required to implement this strategy more broadly.

\section{References}

1. Dake MD, Kato N, Mitchell RS, Semba CP, Razavi MK, Shimono T, et al. Endovascular stent-graft placement for the treatment of acute aortic dissection. N Engl J Med. 1999;340:1546-52.

2. Nienaber CA, Fattori R, Lund G, Dieckmann C, Wolf W, von Kodolitsch Y, et al. Nonsurgical reconstruction of thoracic aortic dissection by stent-graft placement. N Engl J Med. 1999;340:1539-45.

3. Rodriguez JA, Olsen DM, Lucas L, Wheatley G, Ramaiah V, Diethrich EB. Aortic remodeling after endografting of thoracoabdominal aortic dissection. J Vasc Surg. 2008;47:1188-94.

4. Cambria RP, Brewster DC, Lauterbach SR, Kaufman JL, Geller S, Fan CM, et al. Evolving experience with thoracic aortic stent graft repair. J Vasc Surg. 2002;35: 1129-36.

5. Eggebrecht $H$, Nienaber CA, Neuhauser $M$, Baumgart D, Kische $S$, Schmermund A, et al. Endovascular stent-graft placement in aortic dissection: a meta-analysis. Eur Heart J. 2006;27:489-98.

6. Tsai TT, Fattori R, Trimarchi S, Isselbacher E, Myrmel T, Evangelista A, et al. Long-term survival in patients presenting with type B acute aortic dissection: insights from the International Registry of Acute Aortic Dissection. Circulation. 2006; 114:2226-31.

7. Chen X, Huang F, Xu M, Wang L, Jiang Y, Xiao L, et al. The stented elephant trunk procedure combined total arch replacement for Debakey I aortic dissection: operative result and follow-up. Interact Cardiovasc Thorac Surg. 2010;11:594-8.

8. Jakob H, Tsagakis K, Tossios P, Massoudy P, Thielmann M, Buck T, et al. Combining classic surgery with descending stent grafting for acute DeBakey type I dissection. Ann Thorac Surg. 2008;86:95-101.
9. Kato M, Ohnishi K, Kaneko M, Ueda T, Kishi D, Mizushima T, et al. New graft-implanting method for thoracic aortic aneurysm or dissection with a stented graft. Circulation. 1996;94(9 Suppl):II188-93.

10. Pochettino A, Brinkman WT, Moeller P, Szeto WY, Moser W, Cornelius K, et al. Antegrade thoracic stent grafting during repair of acute DeBakey I dissection prevents development of thoracoabdominal aortic aneurysms. Ann Thorac Surg. 2009;88:482-90.

11. Uchida N, Ishihara H, Shibamura H, Kyo Y, Ozawa M. Midterm results of extensive primary repair of the thoracic aorta by means of total arch replacemen with open stent graft placement for an acute type A aortic dissection. $J$ Thorac Cardiovasc Surg. 2006;131:862-7.

12. Moon MR, Dake MD, Pelc LR, Liddell R, Castro LJ, Mitchell RS, et al. Intravascular stenting of acute experimental type B dissections. J Surg Res. 1993;54:381-8.

13. Mossop P, Nixon I, Oakes J, Devine TJ, McLachlan CS. Immediate "total" aortic true lumen expansion in type A and B acute aortic dissection after endovascular aortic endografting and GZSD bare stenting. J Thorac Cardiovasc Surg. 2007; 134:1360-2

14. Mossop PJ, McLachlan CS, Amukotuwa SA, Nixon IK. Staged endovascular treatment for complicated type B aortic dissection. Nat Clin Pract Cardiovasc Med. 2005;2:316-21; quiz 22

15. Hofferberth SC, Foley PT, Newcomb AE, Yap KK, Yii MY, Nixon IK, et al Combined proximal endografting with distal bare-metal stenting for management of aortic dissection. Ann Thorac Surg. 2012;93:95-102.

16. Hofferberth SC, Newcomb AE, Yii MY, Yap KK, Boston RC, Nixon IK, et al Combined proximal stent grafting plus distal bare metal stenting for management of aortic dissection: superior to standard endovascular repair? J Thorac Cardiovasc Surg. 2012;144:956-62.

17. Melissano G, Bertoglio L, Kahlberg A, Baccellieri D, Marrocco-Trischitta MM Calliari F, et al. Evaluation of a new disease-specific endovascular device for type B aortic dissection. J Thorac Cardiovasc Surg. 2008;136:1012-8.

18. Ito N, Tsunoda T, Nakamura M, Iijima R, Matsuda K, Suzuki T, et al. Percutaneous bare Z-stent implantation as an alternative to surgery for acute aortic dissection with visceral ischemia. Catheter Cardiovasc Interv. 2003;58:95-100.

19. Nienaber CA, Kische S, Zeller T, Rehders TC, Schneider H, Lorenzen B, et al. Provisional extension to induce complete attachment after stent-graft placement in type B aortic dissection: the PETTICOAT concept. J Endovasc Ther. 2006;13 738-46.

20. Lombardi JV, Cambria RP, Nienaber CA, Chiesa R, Teebken O, Lee A, et al Prospective multicenter clinical trial (STABLE) on the endovascular treatmen of complicated type B aortic dissection using a composite device design. $J$ Vasc Surg. 2012;55:629-40.e2.

21. Hofferberth SC, Nixon IK, Mossop PJ. Aortic false lumen thrombosis induction by embolotherapy (AFTER) following endovascular repair of aortic dissection. J Endovasc Ther. 2012;19:538-45. 